\title{
Reflexões sobre o papel da Atenção Primária à Saúde na pandemia de COVID-19
}

\author{
Reflections on the role of Primary Health Care in the COVID-19 pandemic \\ Reflexiones sobre el papel de la atención primaria de salud en la pandemia de COVID-19
}

\author{
Maria Teresa Garcia Alves ${ }^{1,2,3}$ [0 \\ ${ }^{1}$ Estratégia Saúde da Família da Prefeitura Municipal de Belo Horizonte, Belo Horizonte, MG, Brasil. \\ ${ }_{2}^{2}$ Programa de Residência Médica do Hospital Odilon Behrens, Belo Horizonte, MG, Brasil. \\ ${ }^{3}$ Sociedade Brasileira de Medicina de Família e Comunidade, Rio de Janeiro, RJ, Brasil.
}

\section{Resumo}

Artigo de opinião com reflexões sobre o papel da Atenção Primária à Saúde (APS) na pandemia de COVID-19 a partir da definição de seus atributos: acesso, longitudinalidade, integralidade, coordenação, orientação familiar, orientação comunitária e competência cultural.

Palavras-chave: Pandemia de COVID-19; Atributos da Atenção Primária à Saúde

\section{Abstract}

Opinion article reflecting on the role of Primary Health Care (PHC) in the COVID-19 pandemic from the definition of its attributes: access, longitudinality, integrality, coordination, family orientation, community orientation and cultural competence.

Keywords: COVID-19 Pandemic; Primary Health Care Attributes.

\section{Resumen}

Artículo de opinión con reflexiones sobre el papel de la Atención Primaria de Salud (APS) en la pandemia COVID-19 a partir de la definición de sus atributos: acceso, longitudinalidad, integralidad, coordinación, orientación familiar, orientación comunitaria y competencia cultural.

Palabras clave: Pandemia COVID-19; Atributos de Atención Primaria de Salud.

Como citar: Alves MTG. Reflexões sobre o papel da Atenção Primária à Saúde na pandemia de COVID-19. Rev Bras Med Fam Comunidade. 2020;15(42):2496 https://doi.org/10.5712/rbmfc15(42)2496

Autor correspondente: Maria Teresa Garcia Alves.

E-mail: mariateresagarciaalves@gmail.com Fonte de financiamento: declaram não haver.

Parecer CEP: não encomendado* Procedência: externa Recebido em: 26/04/2020. Aprovado em: 02/06/2020. 
A pandemia pela doença do novo coronavírus 2019 (COVID-19) atingiu mais de 5 milhões de pessoas em todo o mundo até o momento, segundo dados da Organização Mundial da Saúde (OMS). ${ }^{1}$ O foco do cuidado em nível hospitalar tem sido amplamente debatido tanto por profissionais da saúde, quanto por gestores e também pela mídia. Entretanto, a maior parte das pessoas pode ser cuidada na Atenção Primária à Saúde (APS), ${ }^{2}$ que não deve abrir mão do seu papel estruturante nesse momento.

A APS no Sistema Único de Saúde (SUS) é altamente capilarizada - a quase totalidade dos municípios brasileiros é atendida pela Estratégia Saúde da Família, ${ }^{3}$ refletindo a potência desse nível de atenção. Organizar os serviços de APS para responderem com a máxima efetividade dentro dessa nova realidade é responsabilidade de todos os profissionais, particularmente dos Médicos de Família e Comunidade, tendo em vista as competências de gestão e organização de serviços de saúde esperadas em sua formação. ${ }^{4}$

Para contribuir na reestruturação do trabalho das Equipes de Saúde da Família (ESF), compartilho breves reflexões sobre o papel da APS na pandemia a partir da definição de Starfield ${ }^{5}$ por seus atributos.

\section{ACESSO}

AAPS é a porta de entrada preferencial do sistema de saúde, local de referência onde as pessoas buscam o cuidado quando há uma necessidade. O acesso à APS foi fragilizado na pandemia com as recomendações de isolamento social. As ESF precisam se reinventar nesse momento apropriando-se do teleatendimento ${ }^{6}$ para continuar garantindo esse atributo essencial de forma adequada. A ferramenta Whatsapp, por já ser amplamente utilizada pelas pessoas, representa uma forma simples e abrangente para uma efetiva comunicação das ESF com as pessoas ${ }^{7}$, inclusive nas comunidades de baixa renda que não tem acesso à internet de qualidade. $O$ Whatsapp permite também o envio de mensagens de áudio, o que favorece o contato com pessoas não letradas.

\section{LONGITUDINALIDADE}

Esse atributo leva à construção de uma relação de confiança mútua entre as pessoas e as ESF ao longo do tempo. Os profissionais passam a acumular conhecimentos específicos sobre as pessoas que cuidam, muitos deles dentro do que McWhinney e Freeman ${ }^{8}$ chamam de "conhecimento de nível tácito" - um conhecimento muitas vezes abstrato, não expresso em palavras, que possibilita "ver o universal no particular". Esse conhecimento auxilia imensamente na tomada de decisões clínicas assertivas e ao mesmo tempo personalizadas. No contexto de teleatendimentos, em que há uma maior dificuldade em estabelecer uma comunicação efetiva, esse conhecimento é ainda mais importante, reforçando a necessidade da APS ser a porta de entrada inclusive nessa modalidade.

\section{INTEGRALIDADE}

Esse atributo define a necessidade de organização dos serviços de APS para que esse nível de atenção seja o mais resolutivo possível. Com o cancelamento de várias atividades de rotina dos serviços na pandemia, a integralidade está muito prejudicada. Entretanto, com as consultas domiciliares e os atendimentos à distância pelas ESF, é possível garantir o cuidado às síndromes gripais de forma assertiva ${ }^{9}$ e, especialmente, manter o cuidado dos outros problemas de saúde, respeitando a recomendação de isolamento social sempre que possível. 


\section{COORDENAÇÃO}

A APSé responsável por gerenciar o cuidado, supervisionando as informações vindas de outros níveis de atenção, regulando os fluxos para garantir agilidade à atenção de problemas potencialmente graves e adequando todo esse cuidado à realidade das pessoas. A importância desse atributo fica ainda mais evidente nessa pandemia, quando vários serviços oferecidos pela atenção secundária e terciária foram suspensos, com a necessidade de supervisão e regulação pela APS para garantir o cuidado adequado e oportuno às pessoas.

\section{ORIENTAÇÃO FAMILIAR}

O conhecimento do contexto familiar das pessoas permite às ESF identificar o potencial de cuidado de cada família e também o potencial de ameaça à saúde. Na pandemia, esse atributo contribui para orientações mais individualizadas e assertivas de isolamento domiciliar e cuidado aos doentes. Contribui também na identificação de famílias com relações violentas, o que é particularmente importante tendo em vista os números alarmantes de registros de violência doméstica no contexto do isolamento social ${ }^{10}$, com a necessidade de sensibilização e capacitação das ESF para uma abordagem adequada. ${ }^{11}$

\section{ORIENTAÇÃO COMUNITÁRIA}

As necessidades em saúde das pessoas ocorrem dentro de um contexto social e o conhecimento dessa realidade pelas ESF é fundamental para o planejamento de ações estratégicas, racionalizando a organização dos serviços de APS. No contexto da pandemia, é necessário adaptar as orientações para prevenção da transmissão do vírus entre as pessoas à realidade de cada comunidade, com atenção especial às particularidades das mais vulneráveis, como nas comunidades de favelas e periferias ${ }^{12}$. Ao reorganizarem o serviço, as ESF devem levar em conta se há acesso à internet para o uso preferencial do teleatendimento, se há a necessidade de utilização do transporte público coletivo para o deslocamento das pessoas até ao serviço de saúde, se é possível a realização de consultas domiciliares oportunas. É também necessário que as ESF participem, junto ao serviço social e às organizações comunitárias, da identificação das famílias que mais necessitam de recursos e apoio nesse momento. Os Agentes Comunitários de Saúde (ACS) desempenham um papel muito importante nesse atributo por pertencerem à comunidade e vivenciarem a realidade das pessoas.

\section{COMPETÊNCIA CULTURAL}

Esse atributo representa a capacidade das ESF de comunicarem-se de forma efetiva com as pessoas, adaptando-se às suas características culturais. Esse atributo permite que a comunicação com as pessoas continue efetiva, ainda que através de ferramentas de teleatendimento como o Whatsapp.

\section{OUTROS COMENTÁRIOS}

Para além da adaptação dos serviços fortalecendo os atributos da APS na pandemia, é necessário pensar com urgência na segurança tanto dos pacientes quanto dos profissionais:2,13,14 triagem de todos 
os pacientes antes da entrada nas unidades; uso universal de máscaras faciais e higienização das mãos; adequação do espaço físico das unidades garantindo fluxos separados para pacientes com sintomas respiratórios e sem sintomas respiratórios; atenção especial à segurança da sala de imunizações; desinfecção adequada de todos os espaços da unidade; uso correto e racional dos equipamentos de proteção individual (EPI); rodízio dos profissionais no atendimento aos pacientes com sintomas respiratórios visando reduzir o tempo de exposição ao vírus; previsão de afastamento de profissionais por adoecimento.

Para finalizar essa reflexão, trago trecho do livro Primária ${ }^{15}$ para que não deixemos o essencial da APS de lado justo no momento em que as pessoas encontram-se mais fragilizadas e necessitadas:

"A porta aberta, o cuidado ao longo dos anos, diversos profissionais em um esforço conjunto e coordenado, visando resolver a maioria dos problemas de saúde apresentados, considerando as preferências, o contexto familiar, social e cultural da pessoa em questão, esta é a Primária. O primeiro lugar para se cuidar da saúde. Para se viver mais. Para se viver melhor."

\section{REFERÊNCIAS}

1. World Health Organization (WHO). Coronavirus disease (COVID-19) pandemic - Outbreak situation. Geneva:WHO; 2020; [acesso em 2020 Mai 30]. Disponível em: https://www.who.int/emergencies/diseases/novel-coronavirus-2019?gclid=EAlalQobChMlsvSI4erb6QIV hweRCh3oAAPREAAYASAAEgKgA_D_BwE

2. Ministério da Saúde (BR). Protocolo de manejo clínico do coronavírus (COVID-19) na Atenção Primária à Saúde - Versão 9. Brasília (DF): Ministério da Saúde; 2020; [acesso em 2020 Mai 25]. Disponível em: https://portalarquivos.saude.gov.br/images/pdf/2020/ April/08/20200408-ProtocoloManejo-ver07.pdf

3. Ministério da Saúde (BR). E-Gestor Atenção Básica. Informação e Gestão da Atenção Básica. Brasília (DF): Ministério da Saúde; 2020; [acesso em 2020 Mai 25].Disponível em:https://egestorab.saude.gov.br/paginas/acessoPublico/relatorios/relHistoricoCoberturaAB.xhtml

4. Sociedade Brasileira de Medicina de Família e Comunidade (SBMFC).Currículo baseado em competências para Medicina de Família e Comunidade.Rio de Janeiro(RJ):SBMFC;2020;[acesso em2020 Mai 30].Disponível em:http://www.sbmfc.org.br/wp-content/uploads/ media/Curriculo\%20Baseado\%20em\%20Competencias(1).pdf

5. Starfield B.Atenção Primária:equilíbrio entre necessidades de saúde, serviços e tecnologia.Brasília (DF):UNESCO/Ministério da Saúde;2002.

6. Lei no 13.989, de 15 de abril de 2020 (BR). Dispõe sobre o uso da telemedicina durante a crise causada pelo coronavírus (SARS-CoV-2). Diário Oficial da União, Brasília (DF), 15 abr 2020; [acesso em 2020 Mai 31]; Edição 73: Seção 1: 1.Disponível em: http://www.in.gov. br/ en/web/dou/-/lei-n-13.989-de-15-de-abril-de-2020-252726328

7. Dexpertio. Tutorial de Whatsapp para Centros de Saúde, o guia definitivo. Belo Horizonte (MG): Dexpertio;2020; [acesso em 2020 Mai 25]. Disponível em:http://materiais.dexpertio.com.br/whatsapp-centros-saude

8. McWhinney IR, Freeman TR. Manual de Medicina de Família e Comunidade de McWhinney. 4ª ed. Porto Alegre:Artmed;2017.

9. Greenhalgh T, Koh GCH, Car J. Covid-19: avaliação remota em Atenção Primária à Saúde. Rev Bras Med Fam Comunidade. 2020 Abr;15(42):2461.DOI:https://doi.org/10.5712/rbmfc15(42)2461 DOI: https://doi.org/10.5712/rbmfc15(42)2461

10. Fórum Brasileiro de Segurança Pública.Nota Técnica:violência doméstica durante a pandemia de Covid-19.São Paulo:Oficina 22;2020; [acesso em 2020 Mai 30].Disponível em: http://forumseguranca.org.br/wp-content/uploads/2018/05/violencia-domestica-covid-19-v3.pdf

11. Sociedade Brasileira de Medicina de Família e Comunidade (SBMFC). Abordagem da violência contra a mulher no contexto da COVID 19. Rio de Janeiro (RJ):SBMFC;2020; [acesso em 2020 Mai 31].Disponível em:https://www.sbmfc.org.br/wp-content/uploads/2020/05/ Cartilha-viole\%CC\%82ncia-contra-mulher-para-profissionais.pdf

12. Sociedade Brasileira de Medicina de Família e Comunidade (SBMFC). Orientações para favelas e periferias. Segunda edição revista e ampliada. Rio de Janeiro (RJ): SBMFC; 2020; [acesso em 2020 Jun 05]. Disponível em: https://www.sbmfc.org.br/wp-content/ uploads/2020/05/orientac\%CC\%A70\%CC\%83es-para-favelas-e-periferias_2edic\%CC\%A7a\%CC\%83o_Versa\%CC\%83ofinal.pdf

13. Vitória AM, Campos GWS. Só com APS forte o sistema pode ser capaz de achatar a curva de crescimento da pandemia e garantir suficiência de leitos UTI.São Paulo (SP):Telessaúde - UNIFESP;2020; [acesso em 2020 Mai 25]. Disponível em:https://www.telessaude. unifesp.br/images/downloads/So\%CC\%81\%20APS\%20forte\%20para\%20ter\%20leitos\%20UTI.pdf 
14. Mclntosh K, Hirsch MS, Bloom A. Coronavirus disease 2019 (COVID-19): epidemiology, virology, and prevention. UpToDate.2020 Mai; [Epub ahead of print]; [acesso em 2020 Mai 30]. Disponível em: https://www.uptodate.com/contents/coronavirus-disease-2019-covid19-infection-control-in-health-care-and-home-settings?sectionName=INFECTION\%20CONTROL\%20IN\%20THE\%20HEALTH\%20 CARE\%20SETTING\&topicRef=126981\&anchor=H969410871\&source=see_link\#H969410871

15. Berg GL, Claber I, Pastor R.Primária: o essencial da atenção primária à saúde. Caratinga:FUNEC Editora;2012. 